\title{
Fixed Point Theorems and Asymptotically Regular Mappings in Partial Metric Spaces
}

\author{
Satish Shukla, ${ }^{1}$ Ishak Altun, ${ }^{2}$ and Ravindra Sen ${ }^{1}$ \\ ${ }^{1}$ Department of Applied Mathematics, Shri Vaishnav Institute of Technology and Science, Gram Baroli Sanwer Road, \\ Indore (M.P.), 453331, India \\ ${ }^{2}$ Department of Mathematics, Kirikkale University, Yahsihan, 71450 Kirikkale, Turkey
}

Correspondence should be addressed to Ishak Altun; ishakaltun@yahoo.com

Received 16 April 2013; Accepted 12 May 2013

Academic Editors: P. Amodio, H. J. Ruskin, and B. Tadić

Copyright (C) 2013 Satish Shukla et al. This is an open access article distributed under the Creative Commons Attribution License, which permits unrestricted use, distribution, and reproduction in any medium, provided the original work is properly cited.

The notion of asymptotically regular mapping in partial metric spaces is introduced, and a fixed point result for the mappings of this class is proved. Examples show that there are cases when new results can be applied, while old ones (in metric space) cannot. Some common fixed point theorems for sequence of mappings in partial metric spaces are also proved which generalize and improve some known results in partial metric spaces.

\section{Introduction}

Matthews [1] introduced partial metric spaces as a part of the study of denotational semantics of data flow networks. In partial metric space, the usual metric was replaced by partial metric, with a property that the self-distance of any point may not be zero. In fact, it is widely recognized that partial metric spaces play an important role in constructing models in the theory of computation. Partial metric has applications in the branches of science where the size of data point is represented by its self-distance. The fixed point of a contraction mapping in partial metric space has zero selfdistance; that is, fixed point is a total object. Every metric space is a partial metric space with zero self-distance that is, partial metric spaces are the generalization of metric spaces.

O'Neill [2] generalized the concept of partial metric space a bit further by admitting negative distances. The partial metric defined by O'Neill is called dualistic partial metric. Heckmann [3] generalized it by omitting small self-distance axiom. The partial metric defined by Heckmann is called weak partial metric.
Banach contraction principle ensures the existence and uniqueness of a fixed point of a contractive self-map of metric space and has many applications in applied sciences. The fixed point result of Matthews is the generalization of the following Banach contraction principle.

Let $(X, d)$ be a complete metric space and let $f$ be a selfmap on $X$. If there exists $\lambda \in[0,1)$ such that $d(f x, f y) \leq$ $\lambda d(x, y)$ for all $x, y \in X$, then $f$ has a unique fixed point in $X$.

The fixed point result of Matthews is generalized by several authors for single self map in partial metric spaces (see, e.g., [4-6]). Almost all contractive conditions in these papers imply the asymptotic regularity of the mapping under consideration.

The purpose of this paper is to prove some common fixed point theorems for a sequence of self maps on partial metric spaces and generalize the result of Matthews. The notion of asymptotically regular mapping in partial metric spaces is introduced and a fixed point result for the mappings of this class is also proved. 


\section{Definitions and Preliminaries}

First, we recall some definitions and properties of partial metric spaces.

Definition 1 (see [1]). A partial metric on a nonempty set $X$ is a function $p: X \times X \rightarrow \mathbb{R}^{+}\left(\mathbb{R}^{+}\right.$stands for nonnegative reals) such that for all $x, y, z \in X$

$$
\begin{aligned}
& \text { (p1) } x=y \Leftrightarrow p(x, x)=p(x, y)=p(y, y), \\
& \text { (p2) } p(x, x) \leq p(x, y), \\
& \text { (p3) } p(x, y)=p(y, x), \\
& \text { (p4) } p(x, y) \leq p(x, z)+p(z, y)-p(z, z) .
\end{aligned}
$$

A partial metric space is a pair $(X, p)$ such that $X$ is a nonempty set and $p$ is a partial metric on $X$.

It is clear that if $p(x, y)=0$, then from (p1) and (p2), $x=y$. But if $x=y, p(x, y)$ may not be 0 .

Example 2. Let $p: \mathbb{R}^{+} \times \mathbb{R}^{+} \rightarrow \mathbb{R}^{+}$be defined by $p(x, y)=$ $\max \{x, y\}$, for all $x, y \in \mathbb{R}^{+}$, and then $\left(\mathbb{R}^{+}, p\right)$ is a partial metric space.

Each partial metric on $X$ generates a $T_{0}$ topology $\tau_{p}$ on $X$ which has a base the family of open $p$-balls $\left\{B_{p}(x, \epsilon): x \epsilon\right.$ $X, \epsilon>0\}$, where $B_{p}(x, \epsilon)=\{y \in X: p(x, y)<p(x, x)+\epsilon\}$ for every $x \in X$ and $\epsilon>0$.

Theorem 3 (see [1]). For each partial metric $p: X \times X \rightarrow \mathbb{R}^{+}$ the pair $(X, d)$, where $d(x, y)=2 p(x, y)-p(x, x)-p(y, y)$ for all $x, y \in X$ is a metric space.

Here, $(X, d)$ is called induced metric space, and $d$ is metric induced by $p$.

Throughout this paper, we suppose that $(X, p)$ is induced metric space and $d$ is metric induced by $p$.

Let $(X, p)$ be partial metric space. Then,

(1) a sequence $\left\{x_{n}\right\}$ in $(X, p)$ converges to a point $x \in X$ if and only if $p(x, x)=\lim _{n \rightarrow \infty} p\left(x_{n}, x\right)$,

(2) a sequence $\left\{x_{n}\right\}$ in $(X, p)$ is called Cauchy sequence if there exists $\lim _{n, m \rightarrow \infty} p\left(x_{n}, x_{m}\right)$ and is finite,

(3) $(X, p)$ is said to be complete if every Cauchy sequence $\left\{x_{n}\right\}$ in $X$ converges, with respect to $\tau_{p}$, to a point $x \in$ $X$ such that $p(x, x)=\lim _{n, m \rightarrow \infty} p\left(x_{n}, x_{m}\right)$.

Lemma 4 (see $[1,7])$. Let $(X, p)$ be partial metric space, and then

(a) $\left\{x_{n}\right\}$ is a Cauchy sequence in $(X, p)$ if and only if it is a Cauchy sequence in metric space $(X, d)$.

(b) $(X, p)$ is complete if and only if the metric space $(X, d)$ is complete. Furthermore, $\lim _{n \rightarrow \infty} d\left(x_{n}, x\right)=0$ if and only if

$$
p(x, x)=\lim _{n \rightarrow \infty} p\left(x_{n}, x\right)=\lim _{n, m \rightarrow \infty} p\left(x_{n}, x_{m}\right) .
$$

Definition 5. A self map $f$ on a partial metric space $(X, p)$ is said to be asymptotically regular at a point $x$ in $X$, if

$$
\lim _{n \rightarrow \infty} p\left(f^{n} x, f^{n+1} x\right)=0,
$$

where $f^{n}$ denotes the $n$th iterate of $f$ at $x$.

Note that Banach type contractions, generalized contractions, and so forth are asymptotically regular at every point of space.

Example 6. Let $p: \mathbb{R}^{+} \times \mathbb{R}^{+} \rightarrow \mathbb{R}^{+}$be defined by $p(x, y)=$ $\max \{x, y\}$, for all $x, y \in \mathbb{R}^{+}$, and then $\left(\mathbb{R}^{+}, p\right)$ is a partial metric space. The mapping $f: X \rightarrow X$ is defined by $f x=x / 2$, for every $x \in \mathbb{R}^{+}$. Then $f$ is asymptotically regular at every point of $\mathbb{R}^{+}$.

\section{Fixed Point Theorems}

First, we prove the fixed point result for asymptotically regular mappings.

Theorem 7. Let $(X, p)$ be a complete partial metric space and let $f$ be a self map on $X$, satisfying the following condition:

$$
\begin{aligned}
p(f x, f y) & \\
\leq & a_{1} \phi[\min \{p(x, f x), p(y, f y)\}] \\
& +a_{2} \psi[p(x, f x) \cdot p(y, f y)]+a_{3} p(x, y) \\
& +a_{4}[p(x, f x)+p(y, f y)] \\
& +a_{5}[p(x, f y)+p(y, f x)]
\end{aligned}
$$

for all $x, y \in X$, where $a_{i}=a_{i}(x, y)(i=1,2,3,4,5)$ are nonnegative functions such that for arbitrarily fixed $k>0,0<$ $\lambda<1$ and for all $x, y \in X$

$$
\begin{gathered}
a_{1}(x, y), a_{2}(x, y) \leq k, \\
a_{3}(x, y)+a_{4}(x, y)+2 a_{5}(x, y) \leq \lambda,
\end{gathered}
$$

where $\phi, \psi: \mathbb{R}^{+} \rightarrow \mathbb{R}^{+}$are functions such that $\phi(0)=\psi(0)=$ 0 and $\phi, \psi$ are continuous at 0 . If $f$ is asymptotically regular at some $x_{0} \in X$, and then $f$ has a unique fixed point $u$ in $X$ with $p(u, u)=0$.

Proof. Let $x_{n+1}=f x_{n}$ for every $n \geq 0$. If there exists $n$ such that $x_{n}=x_{n+1}$, then $x_{n}$ is a fixed point of $f$. Suppose that $x_{n+1} \neq x_{n}$ for every $n \geq 0$. We show that $\left\{x_{n}\right\}$ is a Cauchy sequence.

Let $m>n$ denote $p_{n}=p\left(x_{n+1}, x_{n}\right)$ and then from (3) we obtain

$$
\begin{aligned}
p\left(x_{n}, x_{m}\right) & \\
\leq & p\left(x_{n}, x_{n+1}\right)+p\left(x_{n+1}, x_{m+1}\right) \\
& +p\left(x_{m+1}, x_{m}\right)-p\left(x_{n+1}, x_{n+1}\right)-p\left(x_{m+1}, x_{m+1}\right) \\
\leq & p_{n}+p_{m}+p\left(f x_{n}, f x_{m}\right) .
\end{aligned}
$$


Using (3), we obtain

$$
\begin{aligned}
p\left(x_{n}, x_{m}\right) \leq & p_{n}+p_{m}+a_{1} \phi\left[\min \left\{p_{n}, p_{m}\right\}\right]+a_{2} \psi\left[p_{n} \cdot p_{m}\right] \\
& +a_{3} p\left(x_{n}, x_{m}\right)+a_{4}\left[p_{n}+p_{m}\right] \\
& +a_{5}\left[p\left(x_{n}, x_{m+1}\right)+p\left(x_{m}, x_{n+1}\right)\right]
\end{aligned}
$$

where $a_{i}=a_{i}\left(x_{n}, x_{m}\right)$. As

$$
\begin{aligned}
& p\left(x_{n}, x_{m+1}\right)+p\left(x_{m}, x_{n+1}\right) \\
& \leq p\left(x_{n}, x_{m}\right)+p_{m}-p\left(x_{m}, x_{m}\right)+p\left(x_{m}, x_{n}\right) \\
& \quad+p_{n}-p\left(x_{n}, x_{n}\right) \\
& \leq 2 p\left(x_{n}, x_{m}\right)+p_{n}+p_{m} .
\end{aligned}
$$

Therefore from (7), it follows that

$$
\begin{aligned}
p\left(x_{n}, x_{m}\right) & \\
\leq & p_{n}+p_{m}+a_{1} \phi\left[\min \left\{p_{n}, p_{m}\right\}\right]+a_{2} \psi\left[p_{n} \cdot p_{m}\right] \\
& +a_{3} p\left(x_{n}, x_{m}\right)+a_{4}\left[p_{n}+p_{m}\right] \\
& +a_{5}\left[2 p\left(x_{n}, x_{m}\right)+\mathrm{p}_{n}+p_{m}\right] \\
= & \left(1+a_{4}+a_{5}\right)\left(p_{n}+p_{m}\right)+\left(a_{3}+2 a_{5}\right) p\left(x_{n}, x_{m}\right) \\
& +a_{1} \phi\left[\min \left\{p_{n}, p_{m}\right\}\right]+a_{2} \psi\left[p_{n} \cdot p_{m}\right] .
\end{aligned}
$$

Using (4) and (5), we obtain

$$
\begin{aligned}
p\left(x_{n}, x_{m}\right) \leq & (1+\lambda)\left(p_{n}+p_{m}\right)+\lambda p\left(x_{n}, x_{m}\right) \\
& +k \phi\left[\min \left\{p_{n}, p_{m}\right\}\right]+k \psi\left[p_{n} \cdot p_{m}\right] \\
(1-\lambda) p\left(x_{n}, x_{m}\right) \leq & (1+\lambda)\left(p_{n}+p_{m}\right) \\
& +k \phi\left[\min \left\{p_{n}, p_{m}\right\}\right]+k \psi\left[p_{n} \cdot p_{m}\right] .
\end{aligned}
$$

Since $f$ is asymptotically regular at $x_{0}$ and $\phi$ and $\psi$ are continuous at zero, therefore from the above inequality we obtain

$$
\lim _{n, m \rightarrow \infty} p\left(x_{n}, x_{m}\right)=0 .
$$

Hence, $\left\{x_{n}\right\}$ is a Cauchy sequence in $(X, p)$, and by Lemma 4 , it is a Cauchy sequence in $(X, d)$. Using completeness of $(X, p)$ and Lemma 4 , it follows that $(X, d)$ is complete and $\left\{x_{n}\right\}$ converges in $(X, d)$. Thus, $\lim _{n \rightarrow \infty} d\left(x_{n}, u\right)=0$ for some $u \in X$.

Again, from Lemma 4, and (11), we have

$$
p(u, u)=\lim _{n \rightarrow \infty} p\left(x_{n}, u\right)=\lim _{n, m \rightarrow \infty} p\left(x_{n}, x_{m}\right)=0 .
$$

To prove that $f u=u$, let us consider the following inequalities:

$$
\begin{aligned}
p(u, f u) & \leq p\left(u, x_{n+1}\right)+p\left(x_{n+1}, f u\right)-p\left(x_{n+1}, x_{n+1}\right) \\
& \leq p\left(u, x_{n+1}\right)+p\left(f x_{n}, f u\right),
\end{aligned}
$$

$$
\begin{aligned}
p(u, f u) \leq & p\left(u, x_{n+1}\right)+a_{1} \phi\left[\min \left\{p_{n}, p(u, f u)\right\}\right] \\
& +a_{2} \psi\left[p_{n} \cdot p(u, f u)\right]+a_{3} p\left(x_{n}, u\right) \\
& +a_{4}\left[p_{n}+p(u, f u)\right] \\
& +a_{5}\left[p\left(x_{n}, f u\right)+p\left(u, f x_{n}\right)\right]
\end{aligned}
$$

where $a_{i}=a_{i}\left(x_{n}, u\right)$. As

$$
\begin{aligned}
& p\left(x_{n}, f u\right)+p\left(u, f x_{n}\right) \\
& \quad \leq p\left(x_{n}, u\right)+p(u, f u)-p(u, u)+p\left(u, x_{n+1}\right) \\
& \quad=p\left(x_{n}, u\right)+p(u, f u)+p\left(u, x_{n+1}\right) .
\end{aligned}
$$

Therefore, (13) gives

$$
\begin{aligned}
p(u, f u) & \\
\leq & \left(1+a_{5}\right) p\left(u, x_{n+1}\right)+\left(a_{3}+a_{5}\right) p\left(x_{n}, u\right)+a_{4} p_{n} \\
& +\left(a_{4}+a_{5}\right) p(u, f u)+a_{1} \phi\left[\min \left\{p_{n}, p(u, f u)\right\}\right] \\
& +a_{2} \psi\left[p_{n} \cdot p(u, f u)\right] .
\end{aligned}
$$

Using (4) and (5), we obtain

$$
\begin{aligned}
p(u, f u) \leq & (1+\lambda) p\left(u, x_{n+1}\right)+\lambda p\left(x_{n}, u\right) \\
& +\lambda p_{n}+\lambda p(u, f u) \\
& +k \phi\left[\min \left\{p_{n}, p(u, f u)\right\}\right]+k \psi\left[p_{n} \cdot p(u, f u)\right] .
\end{aligned}
$$

From (12) and the above inequality, it follows that

$$
p(u, f u) \leq \lambda p(u, f u)<p(u, f u) .
$$

This contradiction shows that $p(u, f u)=0$, that is, $f u=u$. Thus, $u$ is a fixed point of $f$.

Let $v$ be another fixed point of $f$ and $p(u, v)>0$. From (3), we obtain

$$
\begin{aligned}
p(u, v)= & p(f u, f v) \\
\leq & a_{1} \phi[\min \{p(u, f u), p(v, f v)\}] \\
& +a_{2} \psi[p(u, f u) \cdot p(v, f v)]+a_{3} p(u, v) \\
& +a_{4}[p(u, f u)+p(v, f v)] \\
& +a_{5}[p(u, f v)+p(v, f u)] \\
= & a_{1} \phi[\min \{p(u, u), p(v, v)\}] \\
& +a_{2} \psi[p(u, u) \cdot p(v, v)]+a_{3} p(u, v) \\
& +a_{4}[p(u, u)+p(v, v)]+a_{5}[p(u, v)+p(v, u)] \\
= & a_{1} \phi[0]+a_{2} \phi[0]+a_{3} p(u, v) \\
& +a_{4} p(v, v)+a_{5}[p(u, v)+p(v, u)] \\
\leq & \left(a_{3}+a_{4}+2 a_{5}\right) p(u, v),
\end{aligned}
$$


where $a_{i}=a_{i}(u, v)$. The above inequality with (5) gives

$$
p(u, v) \leq \lambda p(u, v)<p(u, v) .
$$

This contradiction proves uniqueness.

Taking $a_{1}(x, y)=a_{2}(x, y)=1 /(1+p(x, y)), \phi(t)=\psi(t)=$ $\alpha \cdot t, 0<\alpha<1, a_{3}(x, y)=\beta, a_{4}(x, y)=a_{5}(x, y)=0$ in the above theorem, we obtain following corollary.

Corollary 8. Let $(X, p)$ be a complete partial metric space and let $f$ be a self map on $X$, satisfying the following condition:

$$
\begin{aligned}
& p(f x, f y) \\
& \leq \alpha \frac{\min \{p(x, f x), p(y, f y)\}+p(x, f x) \cdot p(y, f y)}{1+p(x, y)} \\
& \quad+\beta p(x, y)
\end{aligned}
$$

for all $x, y \in X$, where $\alpha, \beta$ are nonnegative reals, such that $\alpha<1, \beta<1$. If $f$ is asymptotically regular at some $x_{0} \in X$, then $f$ has a unique fixed point $u$ in $X$ with $p(u, u)=0$.

Again taking $a_{1}(x, y)=a_{2}(x, y)=0$ and $a_{3}(x, y)=$ $\alpha, a_{4}(x, y)=\beta, a_{5}(x, y)=\gamma$ in Theorem 7 , we obtain following corollary.

Corollary 9. Let $(X, p)$ be a complete partial metric space and $f$ be a self map on $X$, satisfying following condition:

$$
\begin{aligned}
p(f x, f y) \leq & \alpha p(x, y)+\beta[p(x, f x)+p(y, f y)] \\
& +\gamma[p(x, f y)+p(y, f x)]
\end{aligned}
$$

for all $x, y \in X$, where $\alpha, \beta, \gamma$ are nonnegative reals, such that $\alpha+\beta+2 \gamma<1$. If $f$ is asymptotically regular at some $x_{0} \in X$, then $f$ has a unique fixed point $u$ in $X$ with $p(u, u)=0$.

Taking $\alpha=\gamma=0$ in above the corollary, we obtain the following result.

Corollary 10. Let $(X, p)$ be a complete partial metric space and let $f$ be a self map on $X$, satisfying the following condition:

$$
p(f x, f y) \leq \beta[p(x, f x)+p(y, f y)]
$$

for all $x, y \in X$, where $0 \leq \beta<1$. If $f$ is asymptotically regular at some $x_{0} \in X$, then $f$ has a unique fixed point $u$ in $X$ with $p(u, u)=0$.

The following example shows that the assumption of asymptotic regularity in above theorems cannot be dropped.

Example 11. Let $X=[0,1]$, then $(X, p)$, where $p(x, y)=$ $\max \{x, y\}$ is a complete partial metric space. Define a self map $f$ on $X$, as follows:

$$
f x= \begin{cases}1 & \text { if } x \in\left[0, \frac{1}{2}\right] \\ \frac{x}{2} & \text { if } x \in\left(\frac{1}{2}, 1\right]\end{cases}
$$

Take $\beta=7 / 10$ then $f$ satisfies the contractive condition of Corollary 10 , but $f$ is not asymptotically regular at any point of $X$, and has no fixed point in $X$.

Results similar to the above corollaries are available in usual metric spaces (see, e.g., [8]). In the following we illustrate the existence of self map which satisfies contractive condition of Corollary 10, in partial metric space but not in usual metric space.

Example 12. Let $X=[0,1]$, then $(X, p)$, where $p(x, y)=$ $\max \{x, y\}$ is a complete partial metric space. Define a self map $f$ on $X$, as follows:

$$
f x= \begin{cases}\frac{x}{2} & \text { if } x \in\left[0, \frac{1}{2}\right) ; \\ \frac{1}{4} & \text { if } x \in\left[\frac{1}{2}, 1\right] .\end{cases}
$$

Note that $f$ satisfies all the conditions of Corollary 10, with $\beta=9 / 10$, and has a unique fixed point $u=0$. But $f$ does not satisfy the contractive condition $d(f x, f y) \leq \beta[d(x, f x)+$ $d(y, f y)]$ for all $x, y \in X$, in usual metric space $(X, d)$ with $d(x, y)=|x-y|$ (which is also the induced metric); for example, if we take $x=1 / 2, y=0$, then there is no $\beta$ such that $0 \leq \beta<1$ and $d(f x, f y) \leq \beta[d(x, f x)+d(y, f y)]$. Therefore, results of usual metric spaces cannot be applied.

Now, we will prove some common fixed point theorems.

Theorem 13. Let $(X, p)$ be a complete partial metric space and $\left\{f_{n}\right\}$ be a sequence of self maps on $X$ satisfying the following.

There exist $a_{1}, a_{2}, a_{3}, a_{4}, a_{5} \in[0,1)$ with $a_{1}+a_{2}+a_{3}+a_{4}+$ $a_{5}<1$ such that for all $x, y \in X$

$$
\begin{aligned}
p\left(f_{i} x, f_{j} y\right) \leq & a_{1} p(x, y)+a_{2} p\left(x, f_{i} x\right)+a_{3} p\left(y, f_{j} y\right) \\
& +a_{4} p\left(y, f_{i} x\right)+a_{5} p\left(x, f_{j} y\right) .
\end{aligned}
$$

Then, all the mappings of sequence $\left\{f_{n}\right\}$ have a unique common fixed point $u$ in $X$ with $p(u, u)=0$.

Proof. Let $x_{0} \in X$ and $x_{n+1}=f_{n+1} x_{n}$ for every $n \geq 0$. We show that $\left\{x_{n}\right\}$ is a Cauchy sequence.

If there exists $k \geq 0$, such that $x_{k}=x_{k+1}$, then from (25) we obtain

$$
\begin{aligned}
& p\left(x_{k+2}, x_{k+1}\right) \\
&= p\left(f_{k+2} x_{k+1}, f_{k+1} x_{k}\right) \\
& \leq a_{1} p\left(x_{k+1}, x_{k}\right)+a_{2} p\left(x_{k+1}, f_{k+2} x_{k+1}\right) \\
&+a_{3} p\left(x_{k}, f_{k+1} x_{k}\right)+a_{4} p\left(x_{k}, f_{k+2} x_{k+1}\right) \\
&+a_{5} p\left(x_{k+1}, f_{k+1} x_{k}\right) \\
&= a_{1} p\left(x_{k+1}, x_{k}\right)+a_{2} p\left(x_{k+1}, x_{k+2}\right)+a_{3} p\left(x_{k}, x_{k+1}\right) \\
&+a_{4} p\left(x_{k}, x_{k+2}\right)+a_{5} p\left(x_{k+1}, x_{k+1}\right) \\
&= a_{1} p\left(x_{k+1}, x_{k+1}\right)+a_{2} p\left(x_{k+1}, x_{k+2}\right)+a_{3} p\left(x_{k+1}, x_{k+1}\right) \\
&+a_{4} p\left(x_{k+1}, x_{k+2}\right)+a_{5} p\left(x_{k+1}, x_{k+1}\right) .
\end{aligned}
$$


As $p\left(x_{k+1}, x_{k+1}\right) \leq p\left(x_{k+2}, x_{k+1}\right)$ we have

$$
p\left(x_{k+2}, x_{k+1}\right) \leq\left(a_{1}+a_{2}+a_{3}+a_{4}+a_{5}\right) p\left(x_{k+2}, x_{k+1}\right),
$$

as $a_{1}+a_{2}+a_{3}+a_{4}+a_{5}<1$, it follows that $p\left(x_{k+2}, x_{k+1}\right)=$ 0 , that is, $x_{k+2}=x_{k+1}$.

Similarly, it can be seen that $x_{k}=x_{k+r}$ for every $r>0$. Thus, $\left\{x_{n}\right\}$ is a Cauchy sequence.

Assume that $x_{n} \neq x_{n+1}$ for every $n \geq 0$. Denote $p_{n}=$ $p\left(x_{n+1}, x_{n}\right)$, then from (25) it follows that

$$
\begin{aligned}
p_{n}= & p\left(f_{n+1} x_{n}, f_{n} x_{n-1}\right) \\
\leq & a_{1} p\left(x_{n}, x_{n-1}\right)+a_{2} p\left(x_{n}, f_{n+1} x_{n}\right)+a_{3} p\left(x_{n-1}, f_{n} x_{n-1}\right) \\
& +a_{4} p\left(x_{n-1}, f_{n+1} x_{n}\right)+a_{5} p\left(x_{n}, f_{n} x_{n-1}\right) \\
= & a_{1} p\left(x_{n}, x_{n-1}\right)+a_{2} p\left(x_{n}, x_{n+1}\right)+a_{3} p\left(x_{n-1}, x_{n}\right) \\
& +a_{4} p\left(x_{n-1}, x_{n+1}\right)+a_{5} p\left(x_{n}, x_{n}\right) \\
\leq & a_{1} p_{n-1}+a_{2} p_{n}+a_{3} p_{n-1}+a_{4} p_{n-1}+a_{4} p_{n}-a_{4} p\left(x_{n}, x_{n}\right) \\
& +a_{5} p\left(x_{n}, x_{n}\right)
\end{aligned}
$$

and so

$$
\left(1-a_{2}-a_{4}\right) p_{n} \leq\left(a_{1}+a_{3}+a_{4}\right) p_{n-1}+\left(a_{5}-a_{4}\right) p\left(x_{n}, x_{n}\right) .
$$

Using, symmetry of $p$, we obtain

$$
\left(1-a_{3}-a_{5}\right) p_{n} \leq\left(a_{1}+a_{2}+a_{5}\right) p_{n-1}+\left(a_{4}-a_{5}\right) p\left(x_{n}, x_{n}\right) .
$$

It follows from (29) and (30) that

$$
\begin{gathered}
\left(2-a_{2}-a_{3}-a_{4}-a_{5}\right) p_{n} \leq\left(2 a_{1}+a_{2}+a_{3}+a_{4}+a_{5}\right) p_{n-1} \\
p_{n} \leq \frac{2 a_{1}+a_{2}+a_{3}+a_{4}+a_{5}}{2-a_{2}-a_{3}-a_{4}-a_{5}} p_{n-1} \\
p_{n} \leq \lambda p_{n-1},
\end{gathered}
$$

where $\lambda=\left(2 a_{1}+a_{2}+a_{3}+a_{4}+a_{5}\right) /\left(2-a_{2}-a_{3}-a_{4}-a_{5}\right)<1$, which implies $p_{n} \leq \lambda p_{n-1} \leq \lambda^{2} p_{n-2} \leq \cdots \leq \lambda^{n} p_{0}$, where $p_{0}=p\left(x_{1}, x_{0}\right)$.

For $m>n$, we obtain

$$
\begin{aligned}
& p\left(x_{n}, x_{m}\right) \\
& \leq p\left(x_{n}, x_{n+1}\right)+p\left(x_{n+1}, x_{n+2}\right)+\cdots+p\left(x_{m-1}, x_{m}\right) \\
& -\left[p\left(x_{n+1}, x_{n+1}\right)+p\left(x_{n+2}, x_{n+2}\right)\right. \\
& \left.\quad+\cdots+p\left(x_{m-1}, x_{m-1}\right)\right]
\end{aligned}
$$

$$
\begin{aligned}
& \leq p_{n}+p_{n+1}+\cdots+p_{m-1} \\
& \leq \lambda^{n} p_{0}+\lambda^{n+1} p_{0}+\cdots+\lambda^{m-1} p_{0} \\
& =\lambda^{n} p_{0}\left(1+\lambda+\lambda^{2}+\cdots \lambda^{m-1}\right) \\
& \leq \frac{\lambda^{n}}{1-\lambda} p_{0} .
\end{aligned}
$$

As $\lambda<1$, it follows that $\lambda^{n} \rightarrow 0$ as $n \rightarrow \infty$, which implies

$$
\lim _{n, m \rightarrow \infty} p\left(x_{n}, x_{m}\right)=0 .
$$

Hence $\left\{x_{n}\right\}$ is a Cauchy sequence in $(X, p)$, and by Lemma 4 , it is a Cauchy sequence in $(X, d)$. Using completeness of $(X, p)$ and Lemma 4 , it follows that $(X, d)$ is complete and $\left\{x_{n}\right\}$ converges in $(X, d)$. Thus $\lim _{n \rightarrow \infty} d\left(x_{n}, u\right)=0$ for some $u \in X$.

Again from Lemma 4, and (33) we have

$$
p(u, u)=\lim _{n \rightarrow \infty} p\left(x_{n}, u\right)=\lim _{n, m \rightarrow \infty} p\left(x_{n}, x_{m}\right)=0 .
$$

To prove that $f_{i} u=u$ for any arbitrary fixed $i \in \mathbb{N}$, let us consider the following inequalities:

$$
\begin{aligned}
p\left(f_{i} u, u\right) & \\
\leq & p\left(f_{i} u, f_{n} x_{n-1}\right)+p\left(f_{n} x_{n-1}, u\right)-p\left(f_{n} x_{n-1}, f_{n} x_{n-1}\right) \\
\leq & p\left(f_{i} u, f_{n} x_{n-1}\right)+p\left(x_{n}, u\right) \\
\leq & a_{1} p\left(u, x_{n-1}\right)+a_{2} p\left(u, f_{i} u\right)+a_{3} p\left(x_{n-1}, f_{n} x_{n-1}\right) \\
& +a_{4} p\left(x_{n-1}, f_{i} u\right)+a_{5} p\left(u, f_{n} x_{n-1}\right)+p\left(x_{n}, u\right) \\
= & a_{1} p\left(u, x_{n-1}\right)+a_{2} p\left(u, f_{i} u\right)+a_{3} p\left(x_{n-1}, x_{n}\right) \\
& +a_{4} p\left(x_{n-1}, f_{i} u\right)+a_{5} p\left(u, x_{n}\right)+p\left(x_{n}, u\right) \\
\leq & \left(a_{1}+a_{3}+a_{4}\right) p\left(x_{n-1}, u\right)+\left(a_{3}+a_{5}+1\right) p\left(x_{n}, u\right) \\
& +\left(a_{2}+a_{4}\right) p\left(u, f_{i} u\right)-\left(a_{3}+a_{4}\right) p(u, u) \\
p\left(u, f_{i} u\right) \leq & \frac{a_{1}+a_{3}+a_{4}}{1-a_{2}-a_{4}} p\left(x_{n-1}, u\right)+\frac{a_{3}+a_{5}+1}{1-a_{2}-a_{4}} p\left(x_{n}, u\right) .
\end{aligned}
$$

Using (34), it follows that $p\left(f_{i} u, u\right)=0$; that is, $f_{i} u=u$. Thus, $u$ is a common fixed point of all the maps of sequence $\left\{f_{n}\right\}$.

Let $v$ be another common fixed point of all the maps of sequence $\left\{f_{n}\right\}$ and $p(u, v)>0$.

For any $n \in \mathbb{N}$, from (25), we obtain

$$
\begin{aligned}
p(u, v)= & p\left(f_{n} u, f_{n} v\right) \\
\leq & a_{1} p(u, v)+a_{2} p\left(u, f_{n} u\right)+a_{3} p\left(v, f_{n} v\right) \\
& +a_{4} p\left(v, f_{n} u\right)+a_{5} p\left(u, f_{n} v\right) \\
= & a_{1} p(u, v)+a_{2} p(u, u)+a_{3} p(v, v) \\
& +a_{4} p(v, u)+a_{5} p(u, v) .
\end{aligned}
$$


Using (p2), we have

$$
p(u, v) \leq\left(a_{1}+a_{2}+a_{3}+a_{4}+a_{5}\right) p(u, v)<p(u, v) .
$$

This contradiction proves uniqueness.

Taking $f_{n}=f$ for every $n \in \mathbb{N}$ in above theorem we obtain following Hardy-Rogers-type result and an improvement to Theorem 2, of Altun et al. [5].

Corollary 14. Let $(X, p)$ be a complete partial metric space. Let $f$ be a self map on $X$, satisfying the following.

There exist $a_{1}, a_{2}, a_{3}, a_{4}, a_{5} \in[0,1)$ with $a_{1}+a_{2}+a_{3}+a_{4}+$ $a_{5}<1$ such that for all $x, y \in X$

$$
\begin{aligned}
p(f x, f y) \leq & a_{1} p(x, y)+a_{2} p(x, f x) \\
& +a_{3} p(y, f y)+a_{4} p(y, f x)+a_{5} p(x, f y) .
\end{aligned}
$$

Then, the $f$ has a unique fixed point $u$ in $X$ with $p(u, u)=0$.

Theorem 15. Let $(X, p)$ be a complete partial metric space. Let $\left\{f_{n}\right\}$ be a sequence of self maps on $X$ satisfying the following.

There exists $a_{1}, a_{2}, a_{3}, a_{4}, a_{5} \in[0,1)$ with $a_{1}+a_{2}+a_{3}+a_{4}+$ $a_{5}<1$ and positive integer $m_{i}$ for each $i \in \mathbb{N}$, such that, for all $x, y \in X$

$$
\begin{aligned}
p\left(f_{i}^{m_{i}} x, f_{j}^{m_{j}} y\right) \leq & a_{1} p(x, y)+a_{2} p\left(x, f_{i}^{m_{i}} x\right) \\
& +a_{3} p\left(y, f_{j}^{m_{j}} y\right) \\
& +a_{4} p\left(y, f_{i}^{m_{i}} x\right)+a_{5} p\left(x, f_{j}^{m_{j}} y\right) .
\end{aligned}
$$

Then all the maps of sequence $\left\{f_{n}\right\}$ have a unique common fixed point $u$ in $X$ with $p(u, u)=0$.

Proof. Note that the sequence $\left\{f_{n}^{m_{n}}\right\}$ satisfies all the conditions of Theorem 13, therefore, all the maps of sequence $\left\{f_{n}^{m_{n}}\right\}$ have a unique common fixed point $u$ in $X$ with $p(u, u)=0$, that is, $f_{i}^{m_{i}} u=u$ for every $i \in \mathbb{N}$. Taking $x=f_{i} u, y=u$ in (39) with the fact that $f_{i}^{m_{i}} f_{i} u=f_{i} f_{i}^{m_{i}} u=f_{i} u$ for every $i \in \mathbb{N}$, we obtain $f_{i} u=u$. Thus, $u$ is a common fixed point of all the maps of sequence $\left\{f_{n}\right\}$. Its uniqueness follows from the fact that $f_{i} u=u$ implies $f_{i}^{m_{i}} u=u$ for every $i \in \mathbb{N}$.

\section{Acknowledgment}

This paper is supported by the Scientific and Technological Research Council of Turkey (TUBITAK) TBAG project $212 \mathrm{~T} 212$.

\section{References}

[1] S. G. Matthews, "Partial metric topology," in Proceedings of the 8th Summer Conference on General Topology and Application, vol. 728 of Annals of the New York Academy of Sciences, pp. 183197, 1994.

[2] S. J. O'neill, "Partial metrics: valuations and domain theory," in Proceedings of the 11th Summer Conference on General Topology and Applications, vol. 806 of Annals of the New York Academy of Sciences, pp. 304-315, 1996.
[3] R. Heckmann, "Approximation of metric spaces by partial metric spaces," Applied Categorical Structures, vol. 7, no. 1-2, pp. 71-83, 1999.

[4] I. Altun and A. Erduran, "Fixed point theorems for monotone mappings on partial metric spaces," Fixed Point Theory and Applications, vol. 2011, Article ID 508730, 10 pages, 2011.

[5] I. Altun, F. Sola, and H. Simsek, "Generalized contractions on partial metric spaces," Topology and Its Applications, vol. 157, no. 18, pp. 2778-2785, 2010.

[6] E. Karapinar, "Weak $\varphi$-contraction on partial metric spaces and existence of fixed points in partially ordered sets," Mathematica Aeterna, vol. 1, no. 3-4, pp. 237-244, 2011.

[7] S. Oltra and O. Valero, "Banach's fixed point theorem for partial metric spaces," Rendiconti dell'Istituto di Matematica dell'Università di Trieste, vol. 36, no. 1-2, pp. 17-26, 2004.

[8] L. Ćirić, "Fixed point theorems for asymptotically regular mappings," Mathematical Communications, vol. 10, pp. 111-114, 2005. 


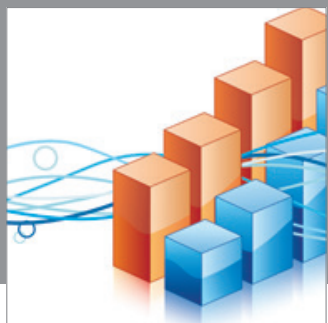

Advances in

Operations Research

mansans

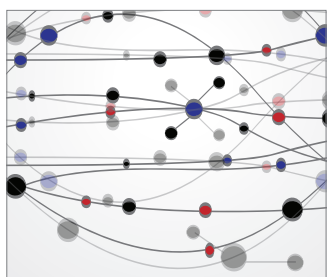

The Scientific World Journal
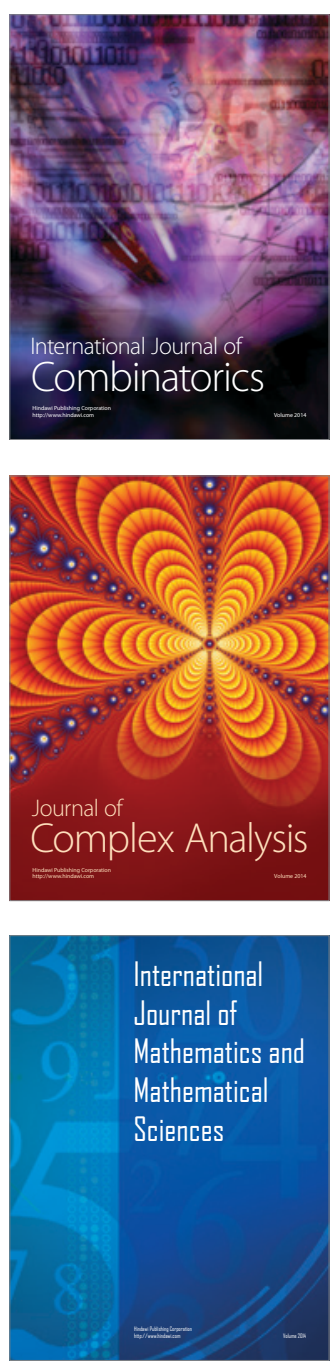
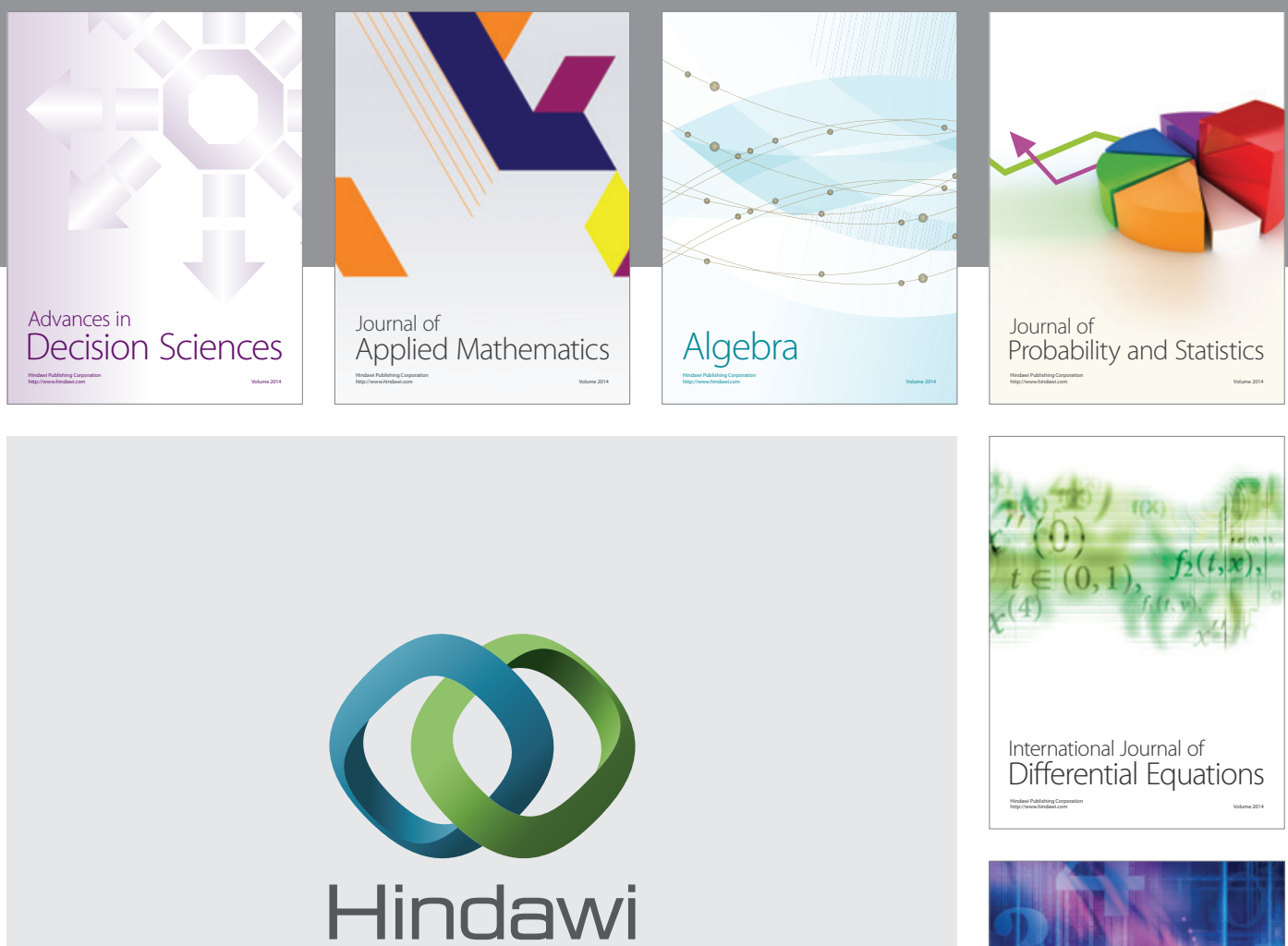

Submit your manuscripts at http://www.hindawi.com
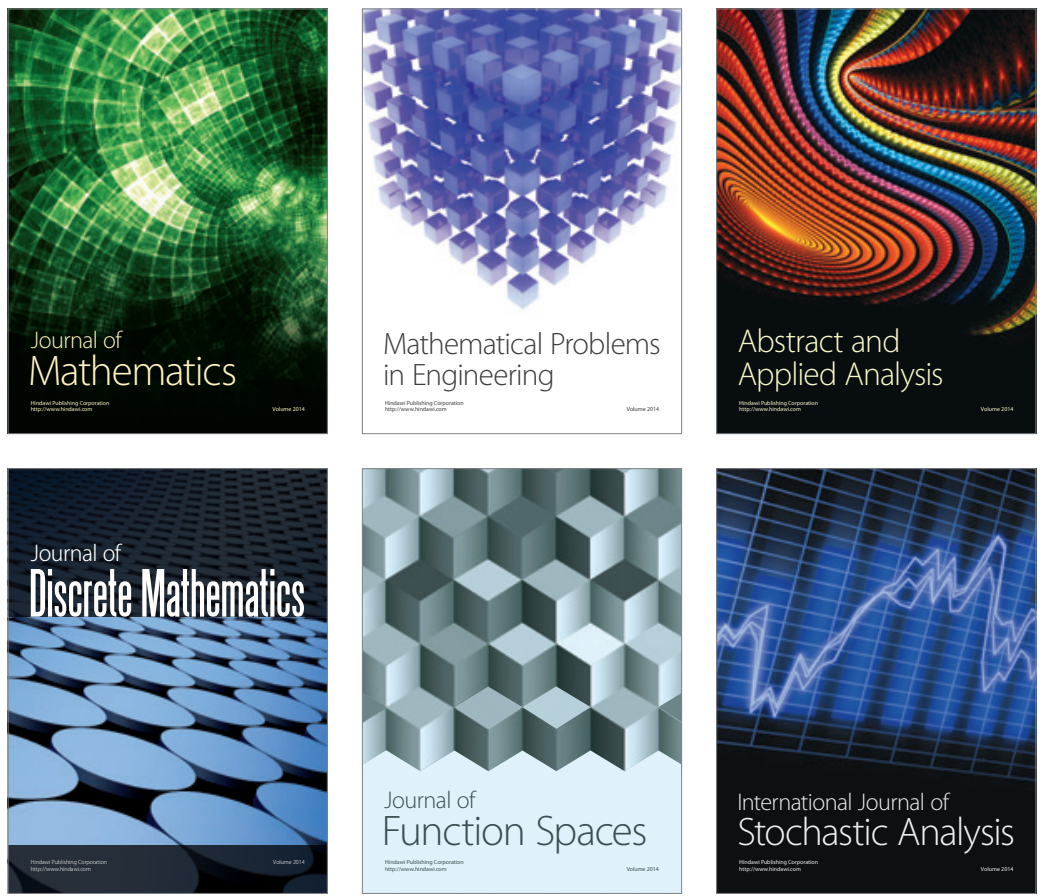

Journal of

Function Spaces

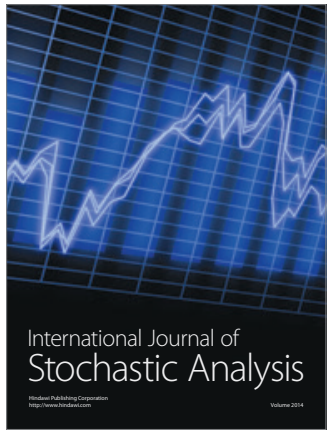

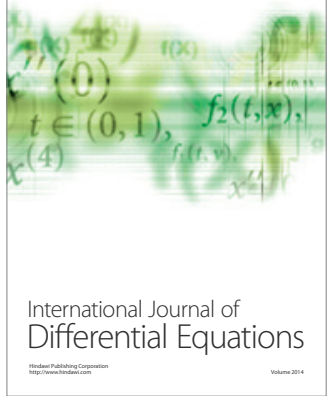
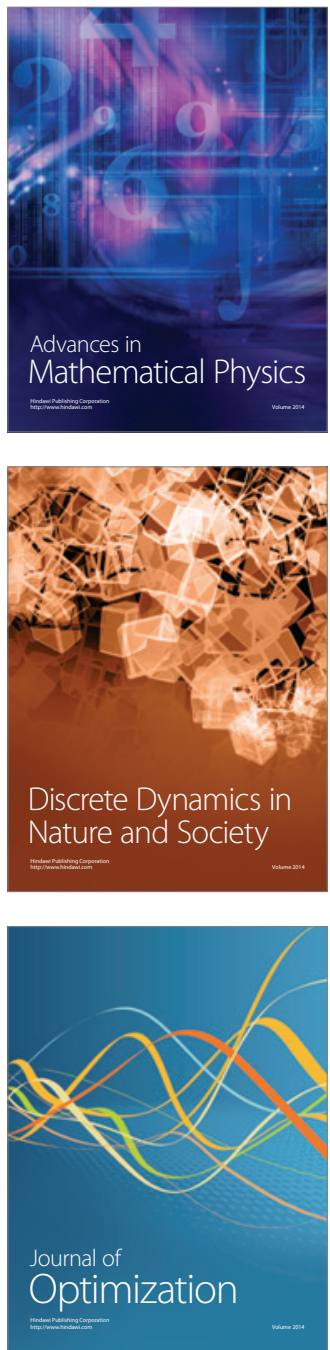